\title{
Nonlinear Model Predictive Control for Pumped Storage Plants Based on Online Sequential Extreme Learning Machine with Forgetting Factor
}

\author{
Chen Feng $\mathbb{D},{ }^{1}$ Chaoshun Li $\left(\mathbb{D},{ }^{2}\right.$ Li Chang, ${ }^{2}$ Zijun Mai, ${ }^{3}$ and Chunwang Wu ${ }^{4}$ \\ ${ }^{1}$ College of Energy and Electrical Engineering, Hohai University, Nanjing 211100, China \\ ${ }^{2}$ College of Civil and Hydraulic Engineering, Huazhong University of Science and Technology, Wuhan 430074, China \\ ${ }^{3}$ Department of Rural Water Management, Nanjing Hydraulic Research Institute, Nanjing 210029, China \\ ${ }^{4}$ NARI Water Conservancy and Hydropower Technology Co., Ltd., Nanjing 211100, China
}

Correspondence should be addressed to Chaoshun Li; csli@hust.edu.cn

Received 11 February 2020; Revised 28 January 2021; Accepted 22 February 2021; Published 5 March 2021

Academic Editor: Peter Giesl

Copyright $(2) 2021$ Chen Feng et al. This is an open access article distributed under the Creative Commons Attribution License, which permits unrestricted use, distribution, and reproduction in any medium, provided the original work is properly cited.

\begin{abstract}
With renewable energy (RE) being increasingly connected to power grids, pumped storage plants (PSPs) play a very important role in restraining the fluctuation of power grids. However, conventional control strategy could not adapt well to the different control tasks. This paper proposes an intelligent nonlinear model predictive control (NMPC) strategy, in which hydraulicmechanical and electrical subsystems are combined in a synchronous control framework. A newly proposed online sequential extreme learning machine algorithm with forgetting factor (named WOS-ELM) is introduced to learn the dynamic behaviors of the coupling system. Specifically, the initial learning parameters are optimized by prior-knowledge learning and a new selfadaptive adjustment strategy is also put forward. Subsequently, the stair-like control strategy and artificial sheep algorithm (ASA) are used in rolling the optimization mechanism to replace the existing complex differential geometric solutions. Comparative experiments are carried out under different working conditions based on a PSP in China. The results show that the influence from coupling factors can be considerable and the proposed MPC strategy indicates superiority in voltage and load adjustment as well as the frequency oscillation suppression.
\end{abstract}

\section{Introduction}

Due to the increasingly serious situation of climate change and air pollution, wind, solar, and other emerging form of renewable energy (RE) have developed rapidly in the past decade $[1,2]$. The international energy agency (IEA) predicts the energy consumption for the next 20 years, with RE accounting for 20\%, as shown in Figure 1. However, RE has the features of intermittent and random fluctuations, and the security and stability of power systems are facing severe challenges $[4,5]$. Pumped storage plants (PSPs) are generally considered to be the most promising technology, which enhances the power grid's ability to absorb RE. They shoulder the important responsibility of power and frequency modulation as well as emergency backup [6].
Therefore, the control quality of the fast response of PSPs is crucial for the utilization efficiency of RE sources and the energy quality of the modern power grid.

Conventional control strategies usually divide the PSP system into hydraulic-mechanical and electrical subsystems, thus facilitating the study of the control problem separately, as shown in Figure 2, which is not conducive to the overall control. (1) Studies of pump-turbine governing control provide a detailed description of the hydraulic-mechanical subsystem while the electrical subsystem is greatly simplified, such as researches on the frequency control of PSP [7-10]. (2) Studies of synchronous generator excitation control are rightly on the contrary. Attention is concentrated on the electrical subsystem, and the dynamics of the hydraulic-mechanical subsystem are ignored. When studying 


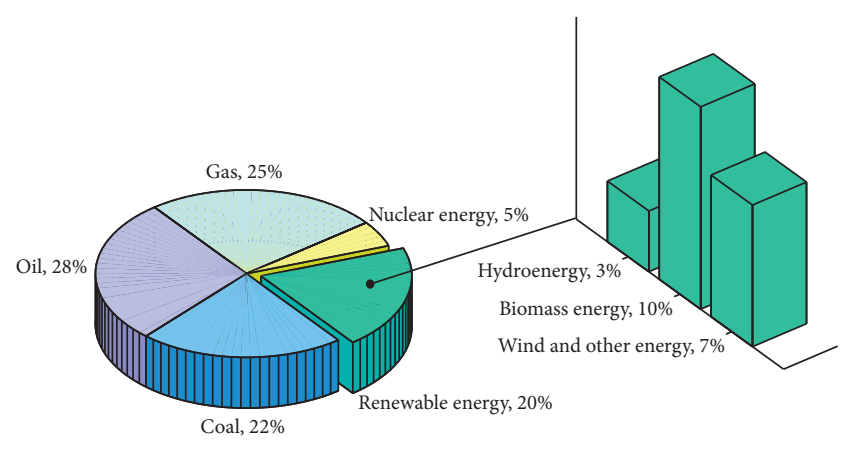

FIgURE 1: IEA/World Energy Outlook 2018/prediction of global energy consumption in 2040 [3].

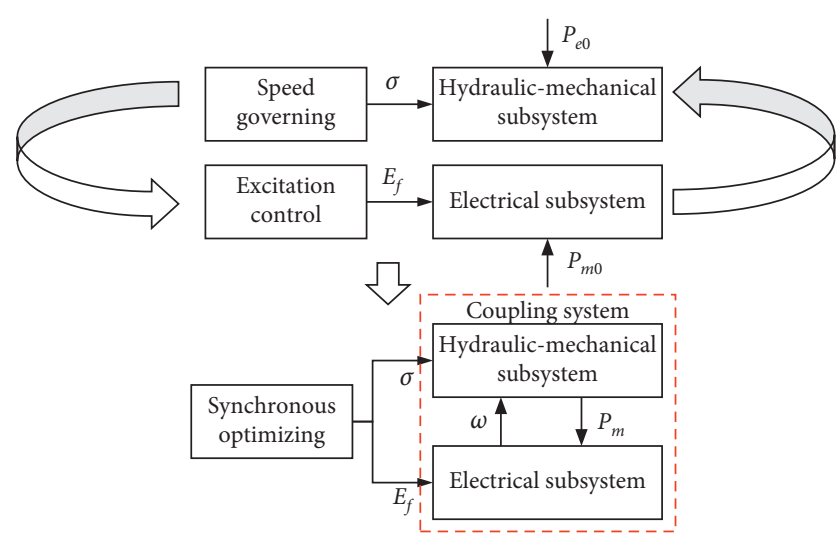

FIGURE 2: Comparison between conventional and proposed control strategy.

the advantages of PSP in mitigating wind power variations [11], a very simple linear model is adopted for pump-turbine. Even more, the mechanical power is considered as a constant in excitation regulation process, which is a standard approach in textbook [12]. The two subsystems have been weakly coupled previously since the response of the hydraulic-mechanical system is relatively slow. But now, demand for flexible and stable regulation of voltage and power frequency by PSP has never been greater [13]. Therefore, hydraulic-mechanical and electrical subsystems should be put into a synchronous control framework. Unfortunately, the coupling models integrated two subsystems and the synchronous control problem have been seldom researched and remained a challenging work.

Although the synchronous control methods about the coupling system of PSPs are rarely studied, a lot of effort has been devoted to improve the dynamic performance and enhance the transient stability of the subsystems separately. The classical Proportional-Integral-Derivative (PID) or PIDtype controllers have been utilized in pump-turbine governing control $[8,14,15]$. At present, the combination of automatic voltage regulator (AVR) and power system stabilizer (PSS) is still the main excitation mode of synchronous generator in engineering practice [16]. However, most of these control strategies have been designed specifically for a certain operating condition and thus cannot automatically adjust control law while there are uncertain tasks or disturbances of environmental effects in the plant. The control strategy may achieve satisfied control effect under one condition but degrade the performance under the other [17]. As a result, theories that can solve the nonlinear control problem with variable conditions are needed to apply in a large complicated power system. As an advanced control strategy, model predictive control (MPC) can solve the timevarying control problem with multivariable interactions. It has been gradually used in the control of PSPs in recent years [18-20], but none of them can adapt to the trend of system descriptions toward nonlinear uncertain models. Recently, with the breakthrough development of artificial intelligence (AI) technology, the concept of MPC has been greatly expanded, and many new MPC design ideas and researches based on artificial neural networks (ANN) have sprung up.

The use of ANN in MPC has grown significantly in various applications over the years [21-24]. Most of these applications are large-scale time control problems calculated in minutes or even hours and employ back-propagation (BP) algorithm to train or update the prediction model. If applied in PSPs, where control laws are often determined within 40 milliseconds, and they may suffer from slow error convergence and can only be trained offline. Hence, the prediction models in these previous studies are not suitable for practical use in PSPs [25]. To overcome the shortcomings of BP, extreme learning machine (ELM), which is a fast machine learning algorithm based on single hidden layer feedforward networks (SLFNs), was proposed by Huang in 2006 [26]. The key features of ELM compared with other famous neural network algorithms are that the parameters in the hidden nodes are generated randomly and the output weights are calculated by the method of least square (LS). In real applications, data are often obtained one by one or chunk by chunk, so the online sequential extreme learning machine (OS-ELM) was proposed in [27] and used in MPC [28,29]. However, OS-ELM does not take time-varying factors into consideration. With the time passing by, more attention should be paid on the new observations, and the old samples to the model should be ignored gradually. Since then, the application of ELM in MPC remains stagnated due to the lack of appropriate model updating mechanism.

Inspired by these ideas, this paper aims to use MPC to deal with complicated coupling control problems of PSP involved with multivariable interactions and strongly nonlinear behavior while the conventional strategies have been designed specifically for a certain working condition and simplified the coupling model. The key of MPC is the prediction model, which is required to predict and update the nonlinear behavior of the plant precisely and quickly. In view of this, a newly proposed online sequential extreme learning machine algorithm with forgetting factor (named WOS-ELM) [30] is applied in MPC for the first time in order to predict and modify the strongly nonlinear behavior of PSP. In addition, a self-adaptive adjustment strategy according to the prediction error for forgetting factor has also been put forward in this paper and a recently proposed artificial sheep algorithm (ASA) is used for optimizing the initial learning parameters of the hidden layer in the WOSELM. In order to avoid complex mathematical derivation 
and reduce the amount of calculation, the ASA and stair-like control strategy have been applied in the rolling optimization. Comparative experiments have been conducted to verify the proposed approach in control under different working conditions.

The main contributions and novelty of this paper are reflected in the following: (1) a detailed nonlinear model, which combines hydraulic-mechanical and electrical subsystems is established to achieve precise simulations of dynamic response in PSP; (2) based on the coupling system, a synchronous control framework has been designed with MPC for the first time to fulfil the coordination of hydraulicmechanical and electrical subsystems; (3) in the MPC control framework, a novel prediction model of ELM with self-adaptive forgetting mechanism is designed, which will promote the prediction accuracy and timeliness.

The remainder of this paper is organized as follows: Section 2 introduces the background knowledge of PSP, WOS-ELM, and ASA. Section 3 describes the proposed approach of the WOS-ELM based NMPC method for PSP. Section 4 presents the details of the experiment arrangements and results. Finally, Section 5 summarizes our conclusions.

\section{Background Knowledge}

2.1. Nonlinear Model of PSP. PSP is a coupling system, which is composed of hydraulic-mechanical and electrical subsystems, as shown in Figure 3. The hydraulic-mechanical subsystem includes servo, conduit system, and a pumpturbine. Guide vane opening adjusts the mechanical output of the pump-turbine. In the electrical subsystem, the exciter generates the excitation voltage of the synchronous generator to sustain its terminal voltage to the given level. The PSP connected to the power grid is simplified as an infinite bus.

2.1.1. Hydraulic-Mechanical Subsystem. The servomechanism is the actuator of the governor, which is used to drive the guide vanes of pump-turbine according to the output signal of the controller. A standard first-order model [31] is adopted for servomechanism, as described by (1). The elastic water column model of the conduit system [8] is adopted and the frictional loss is considered here, as described in (2). The details of all the symbols in PSP are given in the nomenclature.

$$
\begin{aligned}
& \frac{\Delta y}{\Delta \sigma}=\frac{1}{1+T_{y} s}, \\
& \frac{\Delta h}{\Delta q}=\frac{-T_{w} s}{1+(1 / 2) f T_{e} s+(1 / 8) T_{e}^{2} s^{2}} .
\end{aligned}
$$

As the key component of the system, the pump-turbine is of great importance to PSP. At present, the characteristic curves have been extensively used for the nonlinear modelling of pump-turbine. The nonlinear model of pumpturbine based on characteristic curves can be described by

$$
\begin{aligned}
& \left\{\begin{array}{l}
q_{11}=f_{q}\left(y, n_{11}\right), \\
m_{11}=f_{m}\left(y, n_{11}\right), \\
n_{11}=\frac{\omega}{\sqrt{h}}, \\
q_{11}=\frac{q}{\sqrt{h}}, \\
m_{11}=\frac{m_{t}}{h}, \\
P_{m}=m_{t} \omega,
\end{array}\right. \\
& X=\frac{n_{11}}{e^{q_{11}}} .
\end{aligned}
$$

The functions $f_{q}$ and $f_{m}$ represent flow and moment characteristic of the pump-turbine, respectively, as presented in Figure 4. The "Inverted-S" area of the characteristic curves exhibits a multi-valued characteristic, which brings in difficulty for pump-turbine modelling. To overcome this obstacle, a Logarithmic-Curve-Projection (LCP) method [32] is introduced to convert the unit speed $n_{11}$ by logarithmic projection. The transformation is described in (4). The curves obtained by the LCP method are presented in Figure 5. The LCP curves still take $q_{11}$ and $m_{11}$ as the ordinate values. Compared with the Suter or improved Suter method [33], it can greatly simplify the calculation procedure of the pump-turbine model.

2.1.2. Electrical Subsystem. A classical single machine infinite bus power model is adopted for the electrical subsystem. The model of the generator and the network are described by the classical fifth-order model [34], as shown in (5) and (6), respectively. Resistances in the systems are ignored. The transformer and transmission line are simplified as a reactance $\left(X_{s}\right)$.

$$
\begin{aligned}
& \left\{\begin{array}{l}
\dot{\delta}=(\omega-1) \omega_{0}, \\
T_{j} \dot{\omega}=P_{m}-P_{e}, \\
T_{d 0}^{\prime} \dot{E}_{q}^{\prime}=E_{f}-E_{q}^{\prime}-I_{d}\left(X_{d}-X_{d}^{\prime}\right), \\
T_{d 0}^{\prime \prime} \dot{E}_{q}^{\prime \prime}=E_{q}^{\prime}-E_{q}^{\prime \prime}-I_{d}\left(X_{d}^{\prime}-X_{d}^{\prime \prime}\right), \\
T_{q 0}^{\prime \prime} \dot{E}_{d}^{\prime \prime}=-E_{d}^{\prime \prime}+I_{q}\left(X_{q}-X_{q}^{\prime \prime}\right),
\end{array}\right. \\
& \left\{\begin{array}{l}
V_{d}=V_{s} \sin \delta-I_{q} X_{s} \\
V_{q}=V_{s} \cos \delta+I_{d} X_{s} \\
V_{d}=E_{d}^{\prime \prime}+I_{q} X_{q}^{\prime \prime} \\
V_{g}^{2}=V_{d}^{2}+V_{q}^{2} \\
P_{e}=I_{d} V_{d}+I_{d} V_{d} .
\end{array}\right.
\end{aligned}
$$




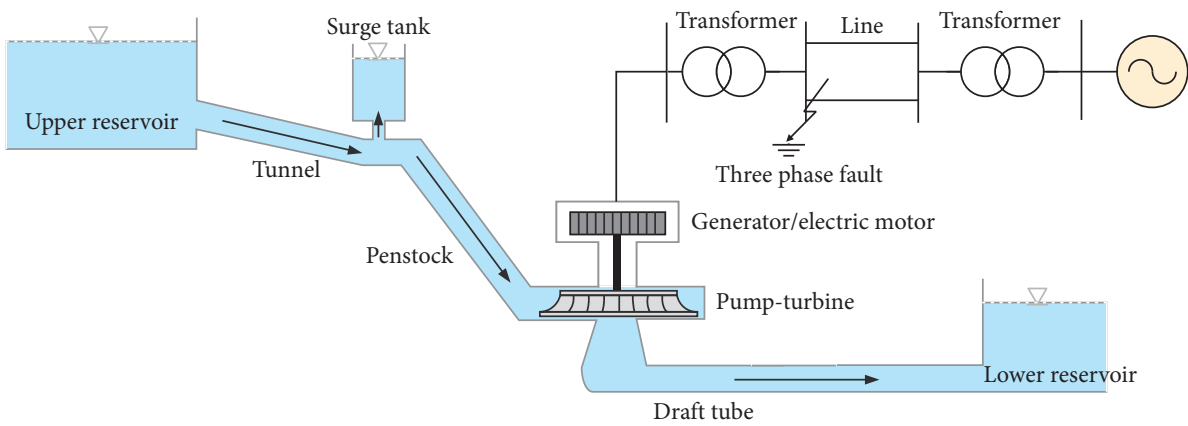

FIGURE 3: Structure of PSP and its interconnected infinite bus.

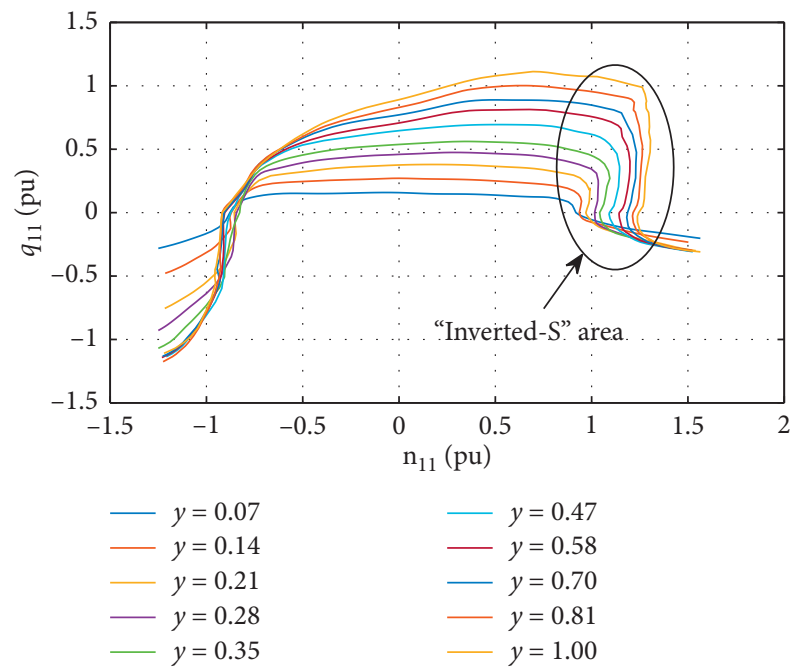

(a)

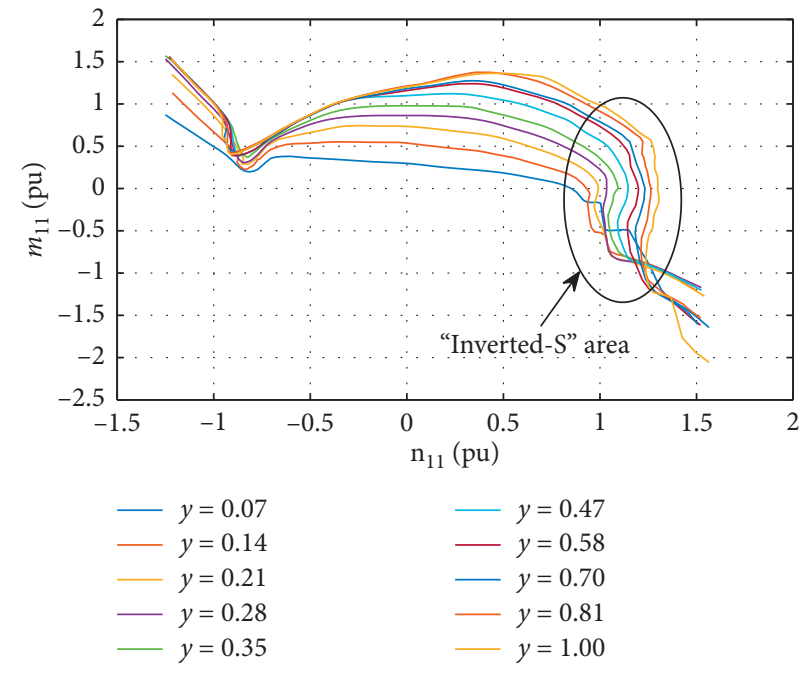

(b)

FIgURE 4: The characteristic curves of a pump-turbine. (a) Flow characteristic curve. (b) Moment characteristic curve.

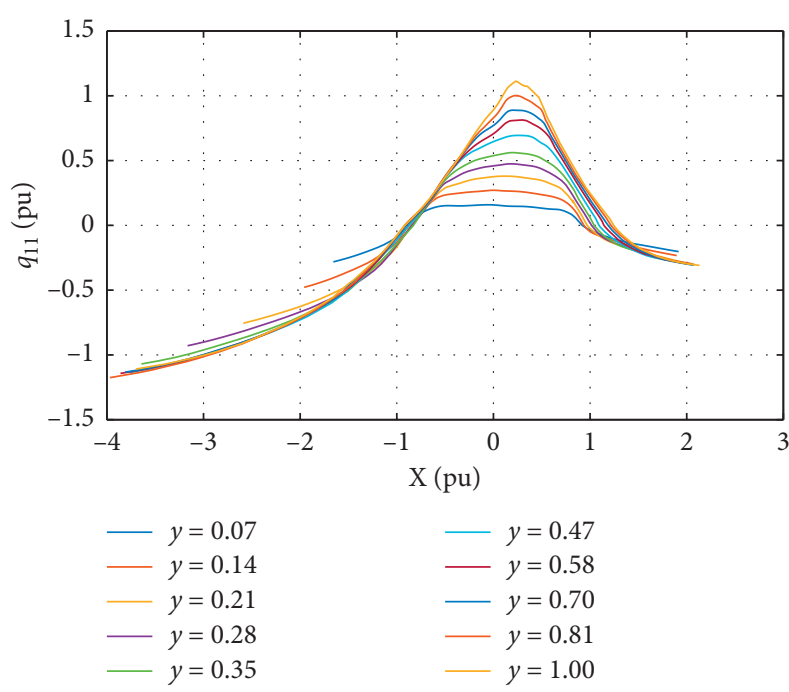

(a)



(b)

FIgURE 5: LCP of the characteristic curves. (a) Flow characteristic curve. (b) Moment characteristic curve. 
2.1.3. Hydraulic-Mechanical-Electrical Coupling System. Based on LPC method and combined with the mathematical models of servomechanism, conduit system, generator, and network, a nonlinear simulation model of PSP is established. The block diagram of the hydraulic-mechanical-electrical coupling system is shown in Figure 6. The proposed NMPC strategy and related experiments in this paper are carried out using this simulation model.

\subsection{Brief Overview of WOS-ELM}

2.2.1. ELM. The ELM shown in Figure 7 is a single hidden layer feedforward network (SLFN), of which input weights and biases of the hidden layer are generated randomly, and the output weights of the hidden layer can be obtained by the theory of least square method [26].

Given the training $\operatorname{set}\left\{\left(x_{i}, t_{i}\right) \mid x_{i} \in R^{D}, t_{i} \in R^{C}, i=1,2\right.$ $, \ldots, N\}$, the SLFN function with $\mathrm{L}$ hidden nodes and an activation function $f(\cdot)$ can be formulated as the following:

$$
\sum_{j=1}^{L} \boldsymbol{\beta}_{j} f\left(\mathbf{w}_{j}, b_{j}, \mathbf{x}_{i}\right)=\mathbf{t}_{i}, \quad i=1,2, \ldots, N,
$$

where $\mathbf{w}_{j}$ and $b_{j}$ are the learning parameters that will be generated randomly; $\beta_{j}$ is the output weight connecting the $j$-th hidden nodes and output nodes; $\mathbf{x}_{i}$ and $\mathbf{t}_{i}$ are the training example and output of the $i$-th observation, respectively. The above $N$ equations can be written into the following form:

$$
\mathrm{H} \boldsymbol{\beta}=\mathrm{T},
$$

where

$$
\mathbf{H}=\left[\begin{array}{ccc}
f\left(\mathbf{w}_{1}, b_{1}, \mathbf{x}_{1}\right) & \ldots & f\left(\mathbf{w}_{L}, b_{L}, \mathbf{x}_{1}\right) \\
\vdots & \ddots & \vdots \\
f\left(\mathbf{w}_{1}, b_{1}, \mathbf{x}_{N}\right) & \ldots & f\left(\mathbf{w}_{L}, b_{L}, \mathbf{x}_{N}\right)
\end{array}\right]_{N \times L},
$$

$\mathbf{H}$ is called the hidden layer output matrix of the network. The coefficient $\beta$ of the ELM can be obtained by solving the least squares solution $\widehat{\beta}$ of the following equation:

$$
\|\mathbf{H} \hat{\beta}-\mathbf{T}\|=\min _{\beta}\|\mathbf{H} \boldsymbol{\beta}-\mathbf{T}\| .
$$

The formula of $\widehat{\beta}$ can be expressed as

$$
\widehat{\beta}=\mathbf{H}^{\dagger} \mathbf{Y}=\left(\mathbf{H}^{T} \mathbf{H}\right)^{-1} \mathbf{H}^{T} \mathbf{T},
$$

where $\mathbf{H}^{\dagger}$ is the Moore-Penrose generalized inverse of $\mathbf{H}$. According to the ridge regression theory, the generalization ability of the ELM and the stability of the network can be enhanced by adding a regularized constant $1 / C$ to the diagonal matrix $\mathbf{H}^{T} \mathbf{H}$. Thus, the final output weight of the ELM can be expressed as follows:

$$
\widehat{\beta}=\left(\mathbf{H}^{T} \mathbf{H}+\frac{1}{C}\right)^{-1} \mathbf{H}^{T} \mathbf{T} .
$$

2.2.2. OS-ELM. In practical applications, the training data may be obtained chunk-by-chunk or one-by-one (a special case of chunk). The OS-ELM algorithm aims at online training and constantly updates the output weights within a short time [27]. When new chunk of sampling data come, the model of ELM should be updated as follows:

$$
\left[\begin{array}{c}
\mathbf{H} \\
\delta \mathbf{H}
\end{array}\right] \beta^{\prime}=\left[\begin{array}{c}
\mathbf{T} \\
\delta \mathbf{T}
\end{array}\right]
$$

where $\delta \mathbf{H}$ and $\delta \mathbf{T}$ are the newly generated hidden layer output and obtained observations, respectively, and $\beta^{\prime}$ is the modified output weight matrix. There are two processes in OS-ELM algorithm, an initialization process and a sequential process. The initialization process is the same as the ordinary ELM algorithm, while the output weight matrix $\beta$ will be updated in the sequential process through an iterative way. The OS-ELM algorithm can be summarized as follows:

Step 1: Initialization. Randomly generate the learning parameters $\mathbf{w}_{j}$ and $b_{j}(j=1,2, \ldots, L)$. Set $k=0$, where $k$ is the index representing the number of chunks of data presented to the ELM. Calculate the initial hidden layer output matrix $\mathbf{H}_{0}$ and the initial output weight $\widehat{\beta}^{(0)}$ by $n_{0}$ sets of initial observations.

$$
\begin{aligned}
\mathbf{H}_{0} & =\left[\begin{array}{ccc}
f\left(\mathbf{w}_{1}, b_{1}, \mathbf{x}_{1}\right) & \ldots & f\left(\mathbf{w}_{L}, b_{L}, \mathbf{x}_{1}\right) \\
\vdots & \ddots & \vdots \\
f\left(\mathbf{w}_{1}, b_{1}, \mathbf{x}_{N_{0}}\right) & \ldots & f\left(\mathbf{w}_{L}, b_{L}, \mathbf{x}_{N_{0}}\right)
\end{array}\right]_{n_{0} \times L}, \\
\hat{\beta}^{(0)} & =\left(\mathbf{H}_{0}^{T} \mathbf{H}_{0}+\frac{1}{C}\right)^{-1} \mathbf{H}_{0}^{T} \mathbf{T}_{0} .
\end{aligned}
$$

Step 2: Calculate the partial hidden layer output matrix $\mathbf{H}_{k+1}$ and the output weight $\widehat{\beta}^{(k+1)}$ by $n_{k+1}$ sets of newly obtained observations.

$$
\begin{aligned}
\mathbf{H}_{k+1}= & {\left[\begin{array}{ccc}
f\left(\mathbf{w}_{1}, b_{1}, \mathbf{x}_{N_{k+1}}\right) & \ldots & f\left(\mathbf{w}_{L}, b_{L}, \mathbf{x}_{N_{k+1}}\right) \\
\vdots & \ddots & \vdots \\
f\left(\mathbf{w}_{1}, b_{1}, \mathbf{x}_{N_{k+1}}\right) & \ldots & f\left(\mathbf{w}_{L}, b_{L}, \mathbf{x}_{N_{k+1}}\right)
\end{array}\right]_{n_{k+1} \times L}, } \\
& \left\{\begin{array}{l}
\hat{\beta}^{(k+1)}=\widehat{\beta}^{(k)}+\mathbf{P}_{k+1} \mathbf{H}_{k+1}^{T}\left(\mathbf{T}_{k+1}-\mathbf{H}_{k+1} \widehat{\beta}^{(k)}\right), \\
\mathbf{P}_{k+1}^{-1}=\mathbf{P}_{k}^{-1}+\mathbf{H}_{k+1}^{T} \mathbf{H}_{k+1},
\end{array}\right.
\end{aligned}
$$

where $\mathbf{P}_{k}$ is the updating matrix, and $\mathbf{P}_{0}=\mathbf{H}_{0}^{T} \mathbf{H}_{0}$.

Step 3: Set $\mathbf{k}=\mathbf{k}+1$, then return to Step 2 .

2.2.3. WOS-ELM. However, OS-ELM does not take timevarying factors into consideration. With the time passing by, the parameters of the model may change. Accordingly, WOS-ELM pays more attention to the new observations and ignores the old samples to the model gradually, which makes the trained model closely track the changes of the system. The updating of the output weight matrix $\beta$ in WOS-ELM is summarized as follows [30]: 




Figure 6: The block diagram of the hydraulic-mechanical-electrical coupling system of PSP.

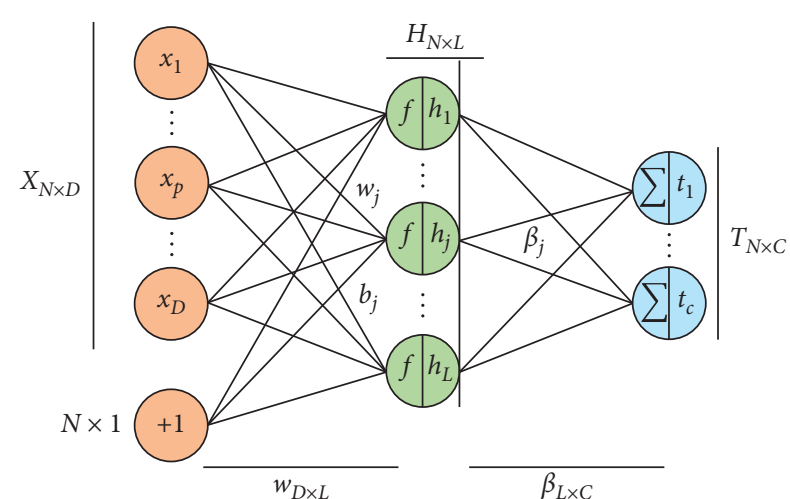

Figure 7: Structure of the ELM.

$$
\left\{\begin{array}{l}
\widehat{\beta}^{(k+1)}=\widehat{\beta}^{(k)}+\mathbf{P}_{k+1} \mathbf{H}_{k+1}^{T}\left(\mathbf{T}_{k+1}-\mathbf{H}_{k+1} \hat{\beta}^{(k)}\right), \\
\mathbf{P}_{k+1}^{-1}=\lambda \mathbf{P}_{k}^{-1}+\mathbf{H}_{k+1}^{T} \mathbf{H}_{k+1},
\end{array}\right.
$$

where $\lambda(0<\lambda \leq 1)$ is the forgetting factor. When $\lambda=1$, WOS-ELM becomes the ordinary OS-ELM algorithm.

2.3. Brief Overview of ASA. The ASA proposed in 2018 is a new intelligent heuristic algorithm, which was inspired by the herd behavior of the sheep flock [35]. The herd individuals move freely within their own neighborhood. Overall, they tend to gather toward the bellwether and follow the natural mechanism of strong and experienced sheep in search of high-quality grassland sources. The algorithm has two main search mechanisms, namely, the strong leading of the bellwether and free strolling of individuals (shown in
Figure 8), which can help to obtain the global optimal solution of high-dimensional and complex problems with a relatively high probability [36].

In ASA, the influence of strong leading of the bellwether is decisive. If the bellwether moves with a big stride, individuals will adjust their motion trajectory $X(t)$ to follow the bellwether closely. The position of the bellwether $X_{B}(t)$ should be recorded and inherited in every time of the iteration. The influence $X_{i}^{b w}(t)$ of the bellwether acting on the i-th sheep $(i=1, \ldots$, popSize $)$ is expressed as follows:

$$
\left\{\begin{array}{l}
\mathbf{X}_{i}^{b w}(t)=\mathbf{X}_{B}(t)+c_{2} \cdot \boldsymbol{\delta}_{i}, \\
\boldsymbol{\delta}_{i}=c_{1} \cdot \mathbf{X}_{B}(t)-\mathbf{X}_{i}(t),
\end{array}\right.
$$

where $\delta_{i}$ is the distance for each individual to the bellwether; $c_{1}=1+(1-\alpha) \cdot \operatorname{rand}_{1} ; c_{2}=2 w \cdot \operatorname{rand}_{1} ; \operatorname{rand}_{1}$ is a random number in $[-1,1] ; \alpha$ is the coefficient of leading scope; $w$ is a dynamic weight decreased from 1 to 0 linearly over the iterations. When strolling or playing, every individual of sheep forage autonomously in a local area, and this behavior is called free strolling of individuals. The shelf-awareness affecting the movement of the $i$-th individual is presented as follows:

$$
\left\{\begin{array}{l}
\mathbf{X}_{i}^{\text {self }}(t)=\mathbf{X}_{i}(t)+\operatorname{rand}_{2} \cdot \boldsymbol{\varepsilon}_{i} \\
\boldsymbol{\varepsilon}_{i}=e^{-\beta \cdot \operatorname{rand}_{1}} \cdot \cos \left(2 \pi \cdot \operatorname{rand}_{1}\right) \cdot \boldsymbol{\delta}_{i}
\end{array}\right.
$$

where rand $_{2}$ is a random number generated from $[0,1]$. Based on the discussion above, the movement of a sheep is affected by the summoning of the bellwether and its free strolling. The position of an individual in an artificial sheep flock will automatically update as follows: 


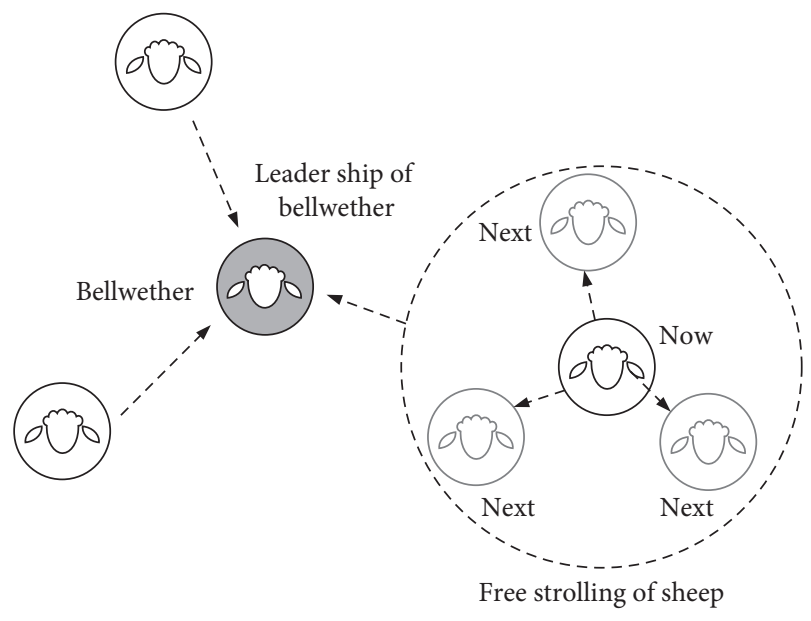

Figure 8: Social behaviors of sheep flock.

$$
\left\{\begin{array}{l}
\mathbf{X}_{i}(t+1)=\varphi_{i} \cdot \mathbf{X}_{i}^{\text {self }}(t)+\left(1+\varphi_{i}\right) \cdot \mathbf{X}_{i}^{b w}(t), \\
\varphi_{i}=w \cdot \operatorname{rand}_{2} .
\end{array}\right.
$$

Published experimental results have shown that ASA could obtain a better global optimal value compared with other algorithms [37]. In this paper, ASA is applied in optimizing the initial learning parameters of the WOS-ELM and the control sequence searching of rolling optimization.

\section{WOS-ELM-Based NMPC}

MPC is mainly composed of three parts: output prediction, rolling optimization, and feedback revision. Its control principle structure is shown in Figure 9. They are coupled to each other to achieve the goal of predicting future state. They can process system constraints explicitly and resist disturbance with uncertain factors, so as to obtain better integrated control performance.

3.1. Output Prediction. One of the basic features of MPC is that the state and output information of the system in the time-domain are predicted by the prediction model of the controlled system based on the known input, output, and state of information of current and historical process. MPC algorithm is not restricted to the form of prediction model. Impulse/step response model, state-space model, time series model, neural network model, and hybrid system model can be applied to the construction of the prediction model. Since MPC has the ability to predict the dynamic behavior of the system in the coming period at each sampling time, researchers can compare the future output trajectory of the system under different control strategies by inputting different control signals into the prediction model.

3.1.1. WOS-ELM Based Output Prediction. With the rapid development of the modern control theory, the neural network model shows obvious advantages in describing the dynamic behavior of complex systems, and becomes the mainstream of system analysis in control theory. Under this

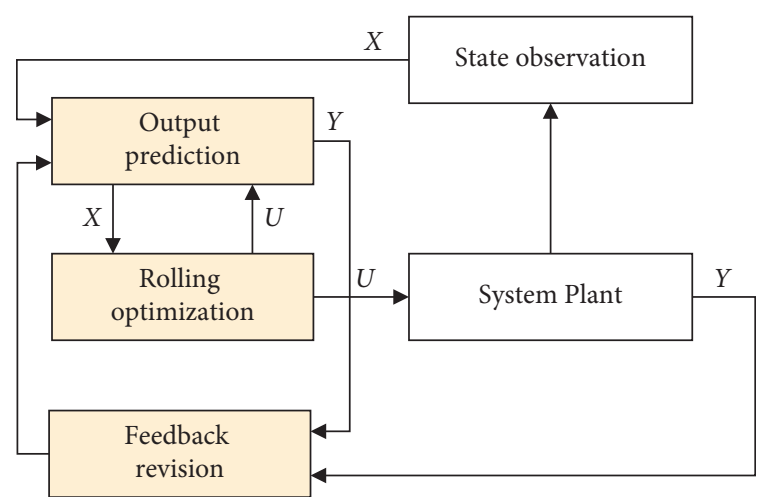

Figure 9: The structure of MPC control system.

background, MPC research using neural network model to describe the system has become a research hotspot in the past decades [28], and has gradually evolved from shallow neural network model to deep neural network model. Since the PSP in the proposed MPC is a MIMO nonlinear system, the model can be expressed by state-space equation of the affine nonlinear system, as shown in

$$
\left\{\begin{array}{l}
\mathbf{x}(k+1)=f(\mathbf{x}(k))+g(\mathbf{x}(k)) \mathbf{u}(k), \\
\mathbf{y}(k)=h(\mathbf{x}(k)) .
\end{array}\right.
$$

The WOS-ELM is selected as the prediction model to predict the output of the PSP. Output prediction process based on WOS-ELM is shown in Figure 10. The state, input, and output variables are given in

$$
\left\{\begin{array}{l}
\mathbf{x}(k)=\left[E_{q}^{\prime}(k), E_{q}^{\prime \prime}(k), E_{d}^{\prime \prime}(k), \delta(k), \omega(k), h(k), y(k)\right], \\
\mathbf{u}(k)=\left[E_{f}(k), \sigma(k)\right], \\
\mathbf{y}(k)=\left[\omega(k), y(k), V_{g}(k)\right] .
\end{array}\right.
$$

Within a prediction horizon $N_{p}$, the state and outputs in several steps in the future can be obtained step-by-step if the input sequences in a prediction horizon are given. The WOS-ELM in kth sampling time remains unchanged, and it is the prediction model of MPC in this paper.

\subsubsection{ASA-Based Prior-Knowledge Learning. Compared} with the traditional BP neural network, the ELM model has faster convergence speed and better generalization performance. However, the input weights and hidden layer biases of the WOS-ELM network are generated randomly, which are relatively independent of the modelling data and cannot effectively reflect the internal relations between the modelling data. In order to obtain the initial weights and the bias of the hidden layer, which can fully reflect the internal relationship of the modelling data, ASA is introduced to search proper input weights and biases. To realize priorknowledge learning, some actual control process data of the system should be obtained in advance. For example, the data of disturbance process controlled by PID, VAR, and PSS can be obtained to estimate the initial parameters. 




FIgURE 10: WOS-ELM based output prediction process in kth sampling time.

The basic idea of the prior-knowledge learning of WOSELM based on ASA can be expressed as: the unknown input weights and hidden layer biases of the WOS-ELM are taken as the decision variables of the optimization algorithm. The output weight can be calculated by (12). The objective is to minimize the deviation between the simulation state variables and the measured values. The optimal parameters are obtained through iterations of the populations of the algorithm. The general structure of the prior-knowledge learning for WOS-ELM based on ASA is presented in Figure 11. The parameters of the WOS-ELM model $\operatorname{are} \theta=[w, b, \beta]$ and a cost function of the mean squared error (MSE) is minimized. It is expressed in (22), where $n$ is the number of learning data.

$$
\operatorname{MSE}(\widehat{\theta})=\frac{1}{n} \sum_{k=1}^{n}\|\mathbf{x}(k)-\widehat{x}(k)\|^{2}
$$

\subsection{Rolling Optimization}

3.2.1. Objective Function. Rolling optimization needs to take output trajectory tracking, control action limitation, as well as terminal state penalty into consideration. It is generally assumed that the state $\mathbf{x}(k \mid k)$ of the system can be measured at time $k$, where the prediction horizon is $N_{p}$. The online optimization problem of MPC is expressed as follows:

$$
J=\sum_{i=0}^{N_{p}-1}\left[\left\|\mathbf{y}(k+i)-\mathbf{y}_{\mathrm{ref}}(k+i)\right\|_{\mathrm{Q}}^{2}+\|\Delta \mathbf{u}(k+i)\|_{R}^{2}\right]+\left[\left\|\mathbf{x}\left(k+N_{p}\right)-\mathbf{x}_{\mathrm{ref}}\left(k+N_{p}\right)\right\|_{P}^{2}\right],
$$

subject to

$$
\begin{aligned}
& \left\{\begin{array}{l}
\mathbf{x}(k+i+1 \mid k)=\widehat{f}(\mathbf{x}(k+i \mid k), \mathbf{u}(k+i \mid k)), \\
\mathbf{y}(k+i \mid k)=\widehat{h}(\mathbf{x}(k+i \mid k)),
\end{array}\right. \\
& \left\{\begin{array}{l}
\mathbf{u} \in\left[\mathbf{U}_{\min }, \mathbf{U}_{\max }\right] \\
\Delta \mathbf{u} \in\left[\Delta \mathbf{U}_{\min }, \Delta \mathbf{U}_{\max }\right],
\end{array}\right.
\end{aligned}
$$

$$
\mathbf{x}\left(k+N_{p}\right)-\mathbf{x}_{\mathrm{ref}}\left(k+N_{p}\right) \in \Omega,
$$

where $Q \in \mathbb{R}^{r \times r}, R \in \mathbb{R}^{m \times m}, P \in \mathbb{R}^{r \times r}$ are the weighting matrices for the output, control input, and terminal state penalty costs, respectively. $\Omega=\left\{\mathbf{x} \mid \mathbf{x}^{T} P \mathbf{x} \leq \alpha\right\}$ is the terminal region for stability. At each sampling time, only the first item of the optimized predictive control law sequence is applied to the system, while at the next sampling time, the online optimization process is repeated. 


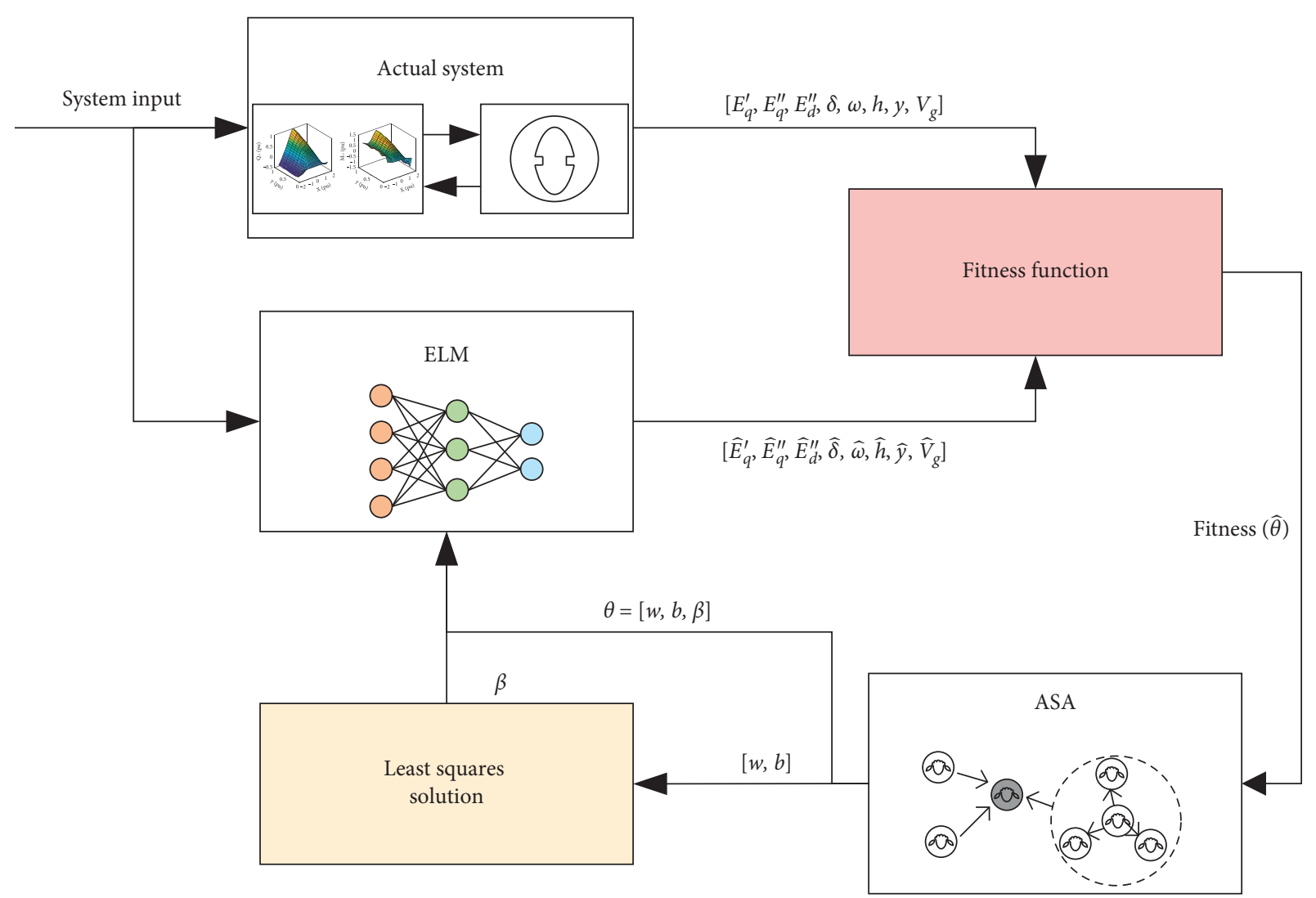

FIGURE 11: The general structure of the prior-knowledge learning for WOS-ELM based on ASA.

3.2.2. ASA-Based Rolling Optimization in MPC. The PSP proposed in MPC is a MIMO nonlinear system, and the rolling optimization belongs to the nonlinear programming with inequality constraints. It is very difficult to obtain the analytical solution of a rolling optimization problem like the linear system. In this paper, a heuristic rolling optimization intelligent solution method based on ASA is proposed.

The excitation system of synchronous generator responds quickly and the transient process is very short. The sampling period of the controller is small (usually $0.01-0.04 \mathrm{~s}$ ), so there is a high requirement for online solving speed of its control law. The rolling optimization of MPC is essentially to solve constrained nonlinear programming online in each sampling period of the controller. According to the optimization theory, the predictive time-domain length of MPC increases and the number of decision variables (i.e. predictive control sequence) to be solved increases. Thus, the corresponding online optimization computation increases exponentially. In order to limit the dimension of decision variables in optimization problems, the idea of stair-like control [38-40] is introduced into model predictive control. By constraining the increment of each step, the trend of change is stair-like.

It is assumed that the control increment in the prediction horizon follows the stair-like rule and changes exponentially in one direction. The stair-like change formulas for controlling increment are shown in (27) and (28).

$$
\frac{\Delta E_{f}\left(k+N_{p} \mid k\right)}{\Delta E_{f}\left(k+N_{p}-1 \mid k\right)}=\cdots=\frac{\Delta E_{f}(k+1 \mid k)}{\Delta E_{f}(k \mid k)}=\mu_{1}\left(\mu_{1} \geq 0\right),
$$

$$
\frac{\Delta \sigma\left(k+N_{p} \mid k\right)}{\Delta \sigma\left(k+N_{p}-1 \mid k\right)}=\cdots=\frac{\Delta \sigma(k+1 \mid k)}{\Delta \sigma(k \mid k)}=\mu_{2}\left(\mu_{2} \geq 0\right),
$$

where $\mu$ is the stair factor. If the stair factor $\mu>1$, the system emphasizes on limiting the change of real-time control quantity and changing the control input in the future. If the stair factor satisfies $0<\mu<1$, the system emphasizes on improving the tracking ability of the system through the action of real-time control quantity, and the closer its value is to 1 , the smoother the whole control process is, but the slower the response is; the closer it is to 0 , the stronger the tracking effect is, but the more the change of the control quantity is.

Once the stair-like control is used in MPC, rolling optimization only needs to search the appropriate value of realtime control variableu $(k \mid k)$, and then the control sequence is calculated by the principle of stair-like control. As a result, the dimension of the optimization problem decreases significantly from $m \times N_{p}$ to $m$, where $m$ is the dimension of the input variables of the system. The lower dimension makes the online optimization much faster. In addition, once the first elements in the control sequence satisfy the 
incremental limit, other incremental constraints in prediction horizon are automatically met.

Since the control increment can be explicitly limited in the ASA search process and the sampling frequency of the system is high, the range of the control signal at each sampling time is relatively small. In the process of solving the control sequence, the population number popsize and the maximal iteration maxGen in ASA can be chosen as a small one. By implementing the above auxiliary operations, the computational complexity of ASA-based rolling optimization process can be significantly reduced.

In each sampling period of the MPC controller, the prediction model iteratively calculates the state and output using the control sequence generated by a sheep in the population. Then, the fitness of each sheep is calculated by the rolling optimization solver according to the cost function in (23). Then, ASA updates the optimal fitness of the population and sets its corresponding coordinates as the current bellwether. The rolling optimization flow based on ASA is shown in Figure 12.

3.3. Feedback Revision. In practice, it is impossible to obtain an accurate model of the object. As a basic prediction model, it is only a rough description of the dynamic characteristics of the object. Because of the uncertainties such as nonlinearity, time-varying, model mismatch, and disturbance in the actual system, the prediction based on the invariant model cannot be exactly consistent with the actual situation, which requires additional prediction methods to supplement the inadequacy of the model prediction, or online correction of the prediction model. Therefore, in each control sampling period, feedback correction mechanism should be introduced into MPC to correct or compensate the state and output prediction errors caused by disturbance or model deviation in real time, so that the whole controlled system becomes a closed-loop control system with feedback mechanism. Only on the basis of feedback revision can rolling optimization show its superiority.

Feedback revision has various forms. It can predict and compensate future errors on the basis of keeping the prediction model unchanged. It can also directly modify the prediction model according to the principle of online identification. Since the WOS-ELM is adopted in this paper, the prediction model can be modified online according to (16), where a suitable value for forgetting factor $\lambda$ plays a significant role in online learning.

When the predicted output agrees well with the newly obtained data, the forgetting factor $\lambda$ should be close to 1 . On the contrary, larger prediction error means the impact of old data becomes weaker. Then, $\lambda$ should tend to $\lambda_{\min }$, which is set in advance. The following adjustment formula (29) can meet the above requirements.

$$
\lambda=\frac{1-\lambda_{\min }}{\pi}\left[-a \tan \left(e_{p}-e_{s}\right)+\frac{\pi}{2}\right]+\lambda_{\min }
$$

where $a$ tan is the arc tangent function, $e_{p}$ is the norm of prediction error, and $e_{s}$ the setting error, as shown in
Figure 13. The forgetting factor can be changed smoothly according to the prediction error.

3.4. Feasibility and Stability. The feasibility of the optimization problem in MPC is that there exists at least one set of control sequences in each sampling period. When they are applied to the prediction model, the states and output trajectories meet all nonlinear constraints (especially terminal inequality constraints) in the system. The feasibility of the MPC algorithm is the precondition of closed-loop stability analysis. A famous lemma about the feasibility of optimization problem is introduced [41].

Lemma 1. For a nonlinear system, ignoring the possible disturbance, the feasibility of an open-loop optimal control problem with equation (23) subject to equations (24)-(26) at time $t=0$ implies the feasibility for $t>0$.

From Lemma 1, if the online optimization of MPC at the initial sampling time is feasible, the feasibility of the whole control process can be guaranteed. The detailed derivation process of this lemma has been described in literature [41], which will not be covered in this paper. The closed-loop stability proof of MPC proposed in this paper is given in detail below.

\section{Theorem 1. Suppose that}

(1) For any $\mathbf{x} \in \Omega$, there exists a control input $\mathbf{K}_{f}(\mathbf{x}) \in \mathbf{U}$ satisfying the inequality (30) [41].

$$
\left\|f\left(\mathbf{x}, \mathbf{K}_{f}(\mathbf{x})\right)\right\|_{P}^{2}-\|\mathbf{x}\|_{P}^{2}+\|\mathbf{x}\|_{Q}^{2}+\left\|\mathbf{K}_{f}(\mathbf{x})\right\|_{R}^{2} \leq 0 .
$$

(2) The open-loop online optimization is feasible at time $t=0$

For a sufficiently small sampling interval, the closed-loop system is asymptotically stable.

Proof. At time $k$, solving the cost function (23), a group of feasible control sequences are obtained as shown in (31). The inputs in this control sequence satisfy the stair-like strategy (27) and (28).

$$
\mathbf{U}^{*}(k)=\left[\mathbf{u}^{*}(k \mid k), \mathbf{u}^{*}(k+1 \mid k), \ldots, \mathbf{u}^{*}\left(k+N_{p}-1 \mid k\right)\right] .
$$

The related state with the control sequence can be presented in

$$
\mathbf{X}^{*}(k)=\left[\mathbf{x}^{*}(k+1 \mid k), \mathbf{x}^{*}(k+2 \mid k), \ldots, \mathbf{x}^{*}\left(k+N_{p} \mid k\right)\right] .
$$

And, the objective function at time $k$ can be obtained in

$$
J^{*}(k)=J\left(k ; \mathbf{U}^{*}(k), \mathbf{X}^{*}(k)\right) .
$$

From the ASA-based optimization mechanism, it can be learned that there exists a control sequence, which makes the 




FIgURE 12: Flow chart of rolling optimization process based on ASA.

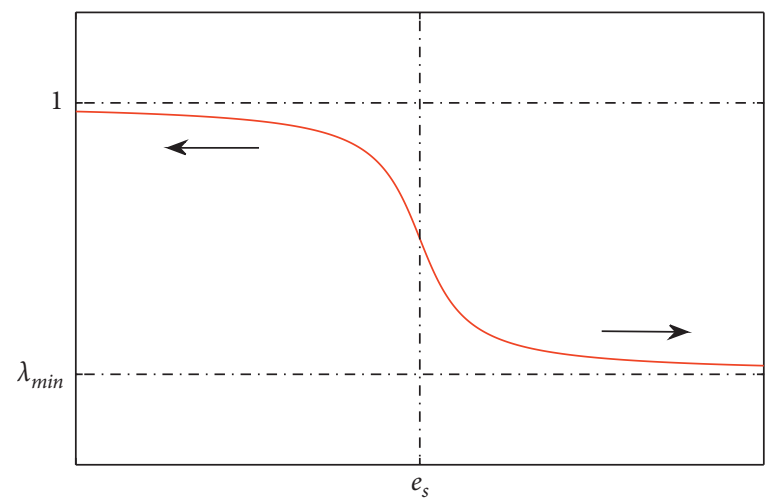

Figure 13: The adjustment function of the forgetting factor. 
relevant part from the optimal solution to lead the state into the terminal region at the next sample time, as shown in

$$
\mathbf{U}(k+1)=\left[\mathbf{u}^{*}(k+1 \mid k), \mathbf{u}^{*}(k+2 \mid k), \ldots, \mathbf{u}^{*}\left(k+N_{p}-1 \mid k\right), \mathbf{K}_{f}\left(\mathbf{x}^{*}\left(k+N_{p} \mid k\right)\right)\right] .
$$

The corresponding state is

$$
\left\{\mathbf{x}^{*}(k+1 \mid k), \ldots, \mathbf{x}^{*}\left(k+N_{p} \mid k\right), f\left(\mathbf{x}^{*}\left(k+N_{p} \mid k\right), \mathbf{K}_{f}\left(\mathbf{x}^{*}\left(k+N_{p} \mid k\right)\right)\right)\right\},
$$

$J^{*}(k+1)$ can be obtained after re-optimizing $J(k+1)$ at time $k+1$. According to the optimality principle, there will exist $J^{*}(k+1) \leq J(k+1)$, and we have

$$
\begin{aligned}
J^{*}(k+1)-J^{*}(k) \leq & J(k+1)-J^{*}(k) \\
= & \left\|f\left(\mathbf{x}^{*}\left(k+N_{p} \mid k\right), \mathbf{K}_{f}\left(\mathbf{x}^{*}\left(k+N_{p} \mid k\right)\right)\right)\right\|_{P}^{2}-\left\|\mathbf{x}^{*}\left(k+N_{p} \mid k\right)\right\|_{P}^{2}+\left\|\mathbf{x}^{*}\left(k+N_{p} \mid k\right)\right\|_{Q}^{2} \\
& +\left\|\mathbf{K}_{f}\left(\mathbf{x}^{*}\left(k+N_{p} \mid k\right)\right)\right\|_{R}^{2}-\left\|\mathbf{x}^{*}(k \mid k)\right\|_{Q}^{2}-\|\mathbf{u}(k \mid k)\|_{R}^{2} .
\end{aligned}
$$

Once the feasible solution exists, there is $\mathbf{x}\left(k+N_{p} \mid k\right) \in \Omega$. Thus, the assumption (1) will result in the inequality

$$
J^{*}(k+1)-J^{*}(k) \leq-\left\|\mathbf{x}^{*}(k \mid k)\right\|_{Q}^{2}-\|\mathbf{u}(k \mid k)\|_{R}^{2}<0 .
$$

Therefore, it can be concluded that $J^{*}(k+1)-J^{*}(k)$ is negative. Because the cost function is quadratic, its value is always positive. Therefore, taking the cost function of rolling optimization in equation (23) as the Lyapunov function of the control system, the closed-loop system is asymptotically stable.

\section{Case Study}

The simulation experiments are mainly divided into three parts, prior-knowledge learning, control performance analysis, and coupling effect analysis. (1) The first part focuses on the prior-knowledge learning of the optimal structure of the ELM model using the normal data set of the voltage regulation process. (2) The second part focuses on the effectiveness and robustness of the proposed MPC strategy. The proposed MPC strategy is compared to conventional PI controller with AVR and PSS, which is designed specifically for a certain operating condition to verify its superiority in the control performance of uncertain conditions. The control structure of PI with AVR and PSS can be referenced in [17]. (3) The third part focuses on the influence of coupling effect on control performance. In this part, two implementations of the proposed MPC strategy are compared. One considers the coupling effect as applied in the second part. The other only takes the electrical subsystem into account but neglects the regulating influence of the hydraulic-mechanical subsystem for its short time span, i.e., guide vane opening control signal $\sigma(t)$ is considered as a constant.

Simulation experiments have been carried out based on the data of PSP in the Jiangxi province of China, as shown in Figure 2. All simulations are implemented using MATLAB 2018a. In order to make the experiment results more convincing and reliable, a sudden step up in terminal voltage, three-phase short circuit fault, and load scheduling cases are selected as the research conditions of robustness analysis. The three-phase short circuit fault case is also selected as the coupling analysis object. The basic parameters and initial steady state of PSP are presented as follows:

(1) System parameters of PSP:

$$
\begin{aligned}
& \text { Generator: } X_{d}=0.768, X_{d}^{\prime}=0.249, X_{d}^{\prime \prime}=0.187, X_{q} \\
& =0.512, X_{q}^{\prime \prime}=0.189, T_{d 0}^{\prime}=5.01, T_{d 0}^{\prime \prime}=0.053, T_{q 0}^{\prime \prime}= \\
& 0.1, T_{j}=7.0
\end{aligned}
$$$$
\text { Transformer and line: } X_{s}=0.3
$$

Servomechanism: $T_{y}=0.2$

Pump-turbine: the characteristic curves are adopted from a real turbine (shown in Figure 3)

Water column: $T_{w}=1.30, T_{e}=0.978, f=0.01$ (the rated water head is $195 \mathrm{~m}$, the rate discharge is $176.1 \mathrm{~m}^{3} / \mathrm{s}$, and the length of the conduit is $L=489.4 \mathrm{~m}$ )

(2) Condition settings of PSP

Initial steady-state condition: $E_{f 0}=1.28, P_{0}=$ $0.9, y_{0}=0.9, \delta_{0}=0.68$ 
Range and rate limitations: $0 \leq \sigma \leq 1,0 \leq E_{f} \leq 3$, $-0.002 \leq \Delta \sigma \leq 0.002,-0.5 \leq \Delta E_{f} \leq 0.5$

(3) Parameter settings of MPC: $N_{p}=50, \mu_{1}=\mu_{2}=$ 0.3 , maxGen $=5$, popSize $=30, \alpha=0, \beta=2, \quad Q=$ $\operatorname{diag}\{1000,1,1\}, R=0, P=\operatorname{diag}\{1,1,1,1,1,1,1\}, \alpha=$ $1.0 \times 10^{-5}, \lambda_{\min }=0.95, e_{s}=1.0 \times 10^{-7}$

4.1. Prior-Knowledge Learning. A normal data set of voltage regulation process is applied for optimizing the optimal structure of the ELM model. The inputs and outputs of ELM have been presented in Section 3, and the optimal number of hidden layer nodes is selected by the minimum MSE between measured outputs and predicted outputs. A tanh nonlinear function is used for the hidden layer, and the transfer function of the output layer is a linear one. The accuracy of ELM models with different number of hidden nodes from 8 to 14 are compared in Table 1 and the numbers of model parameters (input weights, biases, and output weights) are also presented. As the number of hidden layer nodes increases, the MSE decreases while the MSE increases from 11 hidden nodes. Accordingly, the optimal number of hidden layer nodes is chosen as 11. Comparison between the outputs of the ELM model with 11 hidden nodes and the actual system are shown in Figure 14.

4.2. Control Performance Analysis. Most of the control strategies have been designed specifically for a certain operation condition and, thus, cannot automatically identify the change and adjust control law, while there are changes of conditions or environmental disturbances of in the plant. A control strategy may achieve satisfactory control performance under one condition but degrade the performance of the control system under the other. As a result, theories that can solve the nonlinear control problem with uncertain conditions are needed to be applied in a large complicated power system. As an advanced control strategy, MPC can solve the complicated, nonlinear, time-varying control problem with multivariable interactions. In this part, the proposed MPC strategy is compared with conventional PI controller with AVR and PSS to verify its superiority in the control performance of uncertain conditions. In order to obtain satisfactory control performances, the parameters of the PI controller with AVR and PSS are optimized by the ASA with the integral of time multiplied absolute error (ITAE) criterion, as studied in many publications $[14,15]$. The optimized parameters of the PI controller with AVR and PSS are presented in Table 2, where $\mathrm{C} 1$ represents the voltage step condition, C2 represents the three-phase short circuit fault condition, and C3 represents the load adjustment condition. The nomenclature of the parameters is the same as those in [17].

4.2.1. Scenario 1: Sudden Step in Terminal Voltage. $A+10 \%$ sudden step up in terminal voltage is given at $1 \mathrm{~s}$, and simulation results are shown in Figure 15. It is shown that the proposed MPC strategy performs much better than the
TABle 1: Different number of hidden nodes on the complexity and the accuracy of ELM.

\begin{tabular}{lcc}
\hline Hidden nodes & Model parameters & MSE $\left(10^{-7}\right)$ \\
\hline 8 & 144 & 8.7969 \\
9 & 162 & 6.4314 \\
10 & 180 & 1.6794 \\
$\mathbf{1 1}$ & $\mathbf{1 9 8}$ & $\mathbf{0 . 8 0 3 4}$ \\
12 & 216 & 1.0751 \\
13 & 234 & 2.8128 \\
14 & 252 & 6.1366 \\
\hline
\end{tabular}

optimized PI controller with VAR and PSS in voltage response, with nearly no overshoot and oscillation. The rotor speed oscillations of MPC are quicker to calm down. Accordingly, the proposed MPC has presented better performance in voltage regulation and oscillation suppression of the rotor speed, which is beneficial to enhance the stability of the system.

The optimized PI controller with AVR and PSS of two other conditions are also tested in this situation. It is found that the strategy optimized under the other situation could not adapt well to the current operating condition. Despite the optimized conventional strategy of $\mathrm{C} 2$ condition providing a smaller peak-to-peak value of rotor speed, the setting time is much bigger than that of $\mathrm{C} 1$ condition. In addition, the terminal voltage presents a bigger steady-state error. The control performance of the optimized conventional strategy of C3 condition is similar to that of the $\mathrm{C} 1$ condition, but it has a relatively bigger peak-to-peak value and longer setting time in rotor speed and voltage regulation.

4.2.2. Scenario 2: Three-phase Short Circuit Fault. A threephase short circuit fault occurs at the high voltage side of a boost transformer (as shown in Figure 2) at $1 \mathrm{~s}$ and is eliminated after $0.1 \mathrm{~s}$. The comparison results of transition processes between proposed MPC and conventional PI controller with AVR and PSS are given in Figure 16. It can be seen from Figure 16(a) that the proposed MPC provides much stronger damping capability for rotor speed with rapid convergence rates, smaller peak-to-peak value, and less oscillation times. From Figure 16(b), it is shown that the proposed MPC strategy possesses a much quicker voltage response speed with nearly no overshoot.

Similarly, the PI controller with AVR and PSS optimized under this condition is not a halfpenny the worse, which owns good performance in response to rotor speed and terminal voltage. However, in order to ensure the control quality under normal working conditions $(\mathrm{C} 1$ and $\mathrm{C} 3$ conditions), the parameters of PI controller with VAR and PSS are not specifically designed for a three-phase short circuit fault condition. In other words, the control parameters of normal working conditions are applied when a three-phase short circuit fault occurs. It can be found from Figure 16 that the parameters of the normal situation could not adapt well to the fault condition, which can easily lead to low frequency oscillations in the power system. On the other 

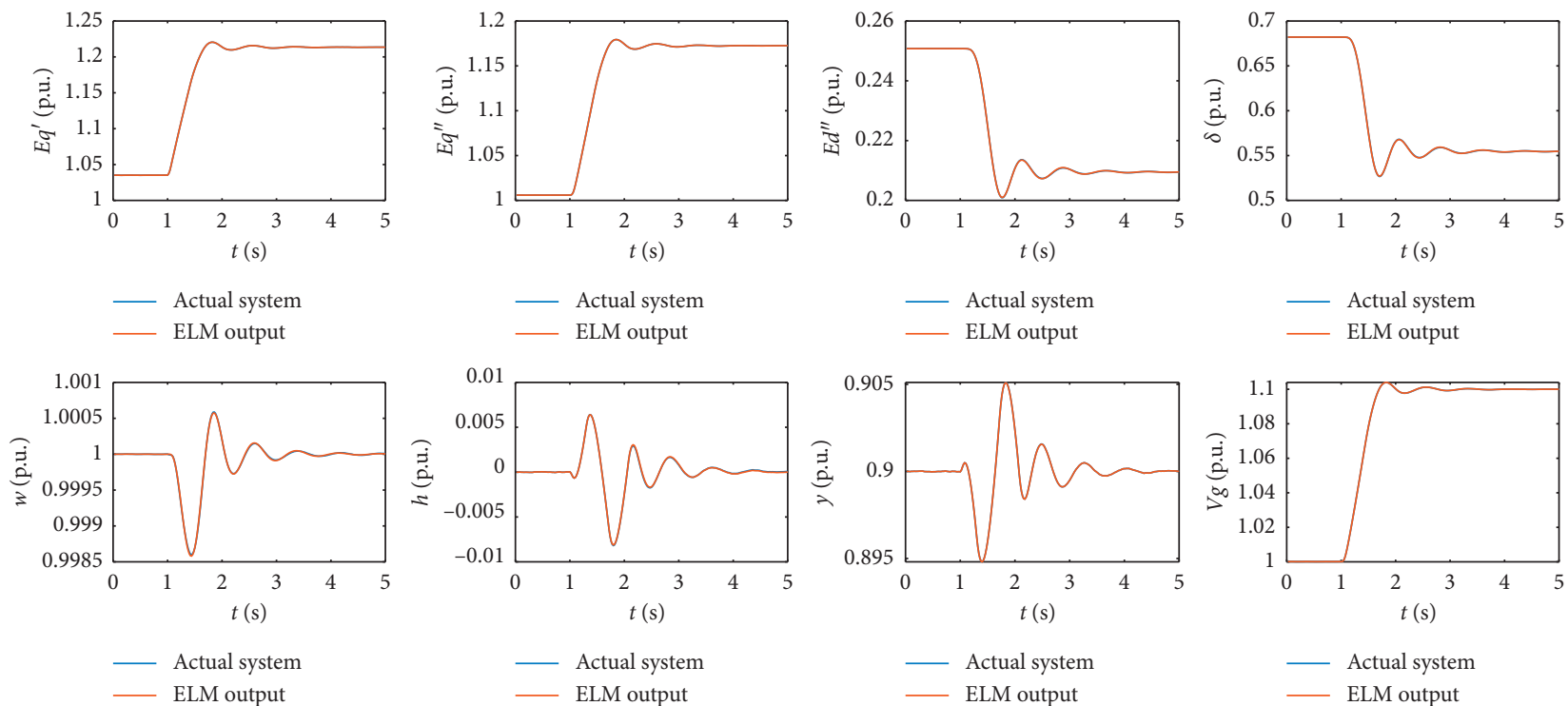

FIGURE 14: Comparison between the outputs of the ELM model and the actual system.

TABLE 2: The optimized parameters of the PI controller with AVR and PSS under different conditions.

\begin{tabular}{lcccccccc}
\hline Condition & $K_{a}$ & $T_{r}$ & $K_{s}$ & $T_{0}$ & $T_{1}$ & $T_{2}$ & $K_{p}$ \\
\hline C1 & 292.81 & 0.006 & 19.02 & 4.63 & 0.34 & 0.252 & 1.67 \\
C2 & 37.10 & 0.017 & 14.05 & 1.38 & 0.91 & 0.005 & 0.21 \\
C3 & 207.85 & 0.015 & 12.79 & 1.46 & 0.70 & 0.415 & 9.83 & 7.71 \\
\hline
\end{tabular}

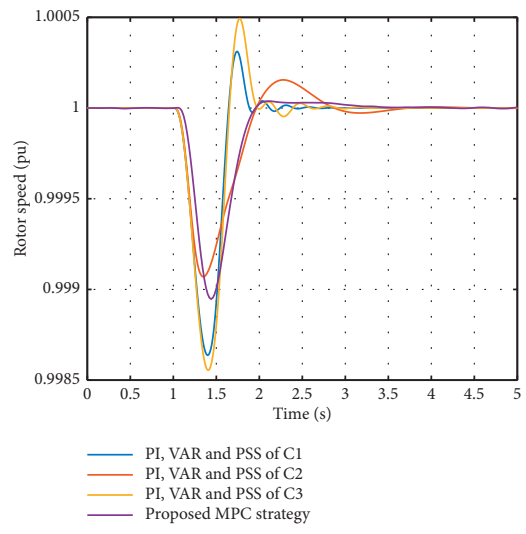

(a)

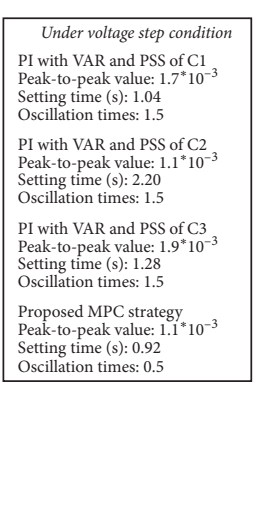
PI with VAR and PSS of C1 Setting time (s): 1.04 PI with VAR and PSS of C2 Seatting time (s): 2.20 PI with VAR and PSS of C3 Peak-to-peak value: $1.9^{*} 10^{-3}$ Oscillation times: 1.5 roposed MPC strategy tting time $(s): 0.92$ scillation times: 0.5

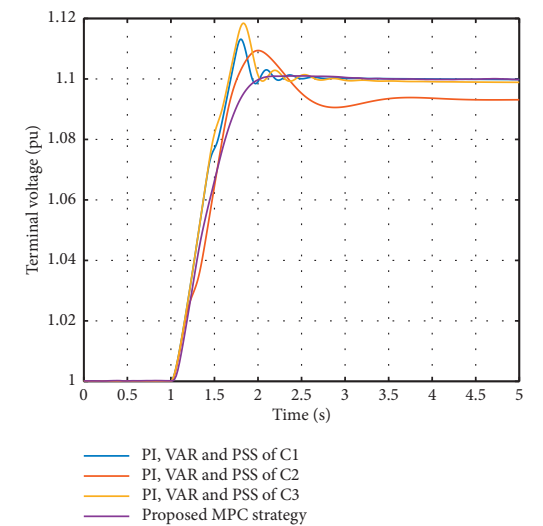

\begin{tabular}{|l|}
\hline Under voltage step condition \\
PI with VAR and PSS of $\mathrm{C} 1$ \\
Overshoot (\%): 13 \\
Setting time $(\mathrm{s}): 1.24$ \\
Oscillation times: 2 \\
PI with VAR and PSS of C2 \\
Overshoot $(\%): 9$ \\
Setting time $(\mathrm{s}):>5$ \\
Oscillation times: 1 \\
PI with VAR and PSS of C3 \\
Overshoot (\%): 18 \\
Setting time $(\mathrm{s}): 1.41$ \\
Oscillation times: 2 \\
Proposed MPC strategy \\
Overshoot (\%): 0 \\
Setting time $(\mathrm{s}): 0.94$ \\
Oscillation times: 0 \\
\hline
\end{tabular}

(b)

Figure 15: Response comparison under terminal voltage step condition. (a) Rotor speed response. (b) Terminal voltage response.

hand, if the control parameters designed for three-phase short circuit fault are used in normal conditions, the control quality of the system cannot be guaranteed (seen Figures 15 and 17). At this point, the advantages of the proposed MPC strategy are prominent for its good adaptability and flexibility in varied conditions.

4.2.3. Scenario 3: Load Adjustment. The load starts to adjust from $90 \%$ to $85 \%$ at 1 s, and the results of the load adjustment process are shown in Figure 17. It can be seen that the guide vane opening strictly follows the objective function while the electrical power has an obvious anti-regulation at the beginning, which is caused by the water hammer effect. Seen from Figure 17, the proposed MPC strategy not only provides a smaller anti-regulation than the PI with VAR and PSS optimized under load adjustment condition but it also has a rapid load response speed and, thus, can undertake the task of fast adjustments on load when a large-scale RE is connected to the grid. 


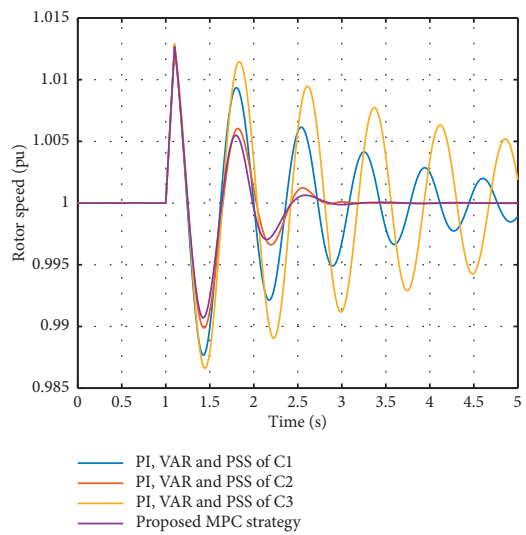

(a)


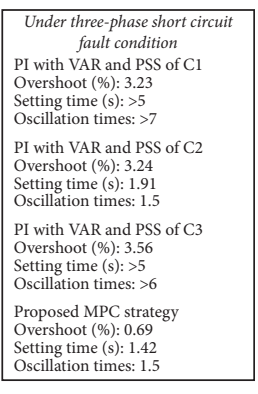

(s)

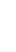

(b)

FIGURE 16: Response comparison under a three-phase short circuit fault condition. (a) Rotor speed response. (b) Terminal voltage response.

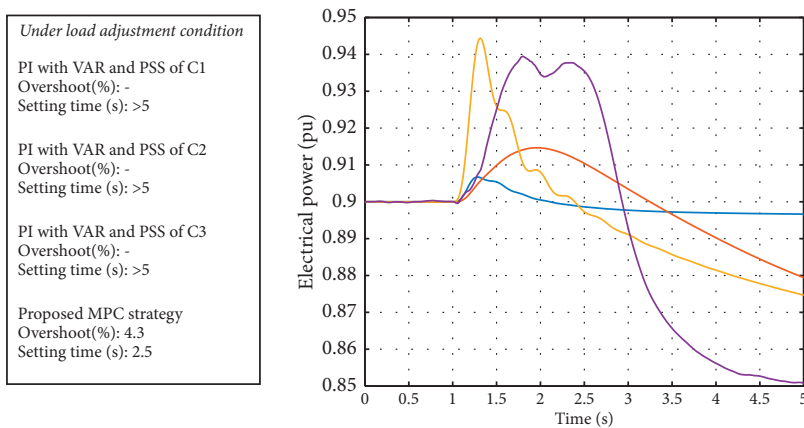

Under load adjustment condition

PI with VAR and PSS of C1 Anti-regulation(\%): 13.43 Setting time $(s):>5$

PI with VAR and PSS of C2 Anti-regulation(\%): 29.30 Setting time $(s):>5$

PI with VAR and PSS of C3 Anti-regulation(\%): 88.73

Proposed MPC strateg Proposed MPC strategy
Anti-regulation $(\%): 78.97$ Setting time (s): 4

Figure 17: Response comparison under load adjustment condition.

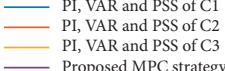

(b)

The PI controller with AVR and PSS optimized under this condition performs a little better than those optimized under the other condition, but it cannot meet the requirements of rapid load scheduling, which is caused by the inherent defects of the PI controller. Setting larger PI parameters may increase the response rate of load adjustment, but it is easy to cause low frequency oscillations in the power system (seen from Figure 16) when the system is subjected by external disturbances. This is the contradiction that the conventional PI with VAR and PSS control methods has to face, but the proposed MPC strategy can easily solve this contradiction due to its local optimization capability.

4.3. Coupling Effect Analysis. The influence of coupling factors on control performance is studied in this part. Two implementations of the proposed MPC strategy are compared. One implementation considers the coupling effect of hydraulic-mechanical-electrical system, as applied in Section 4.2. The other only takes the electrical subsystem into account but neglects the regulating influence of hydraulic-mechanical subsystem for its short time span, i.e., guide vane opening control signal $\sigma(t)$ is considered as a constant, as shown in Figure 18 [38].

The common ranges of some basic time constants in PSP are shown in Figure 19. Three time constants affecting electromagnetic power are marked in red and the other three time constants affecting mechanical power are shown in green. Generally, time constants $T_{d 0}^{\prime \prime}$ and $T_{q 0}^{\prime \prime}$ are very small, the influence of which on the coupling effect of the PSP system can be ignored. Therefore, time constant $T_{d 0}^{\prime}$ is selected as the study factor. Parameter settings of the noncoupling strategy are the same as the coupling strategy. The three-phase short circuit fault is selected as the study condition.

The response comparisons under the three-phase short circuit fault of different transient constant times 


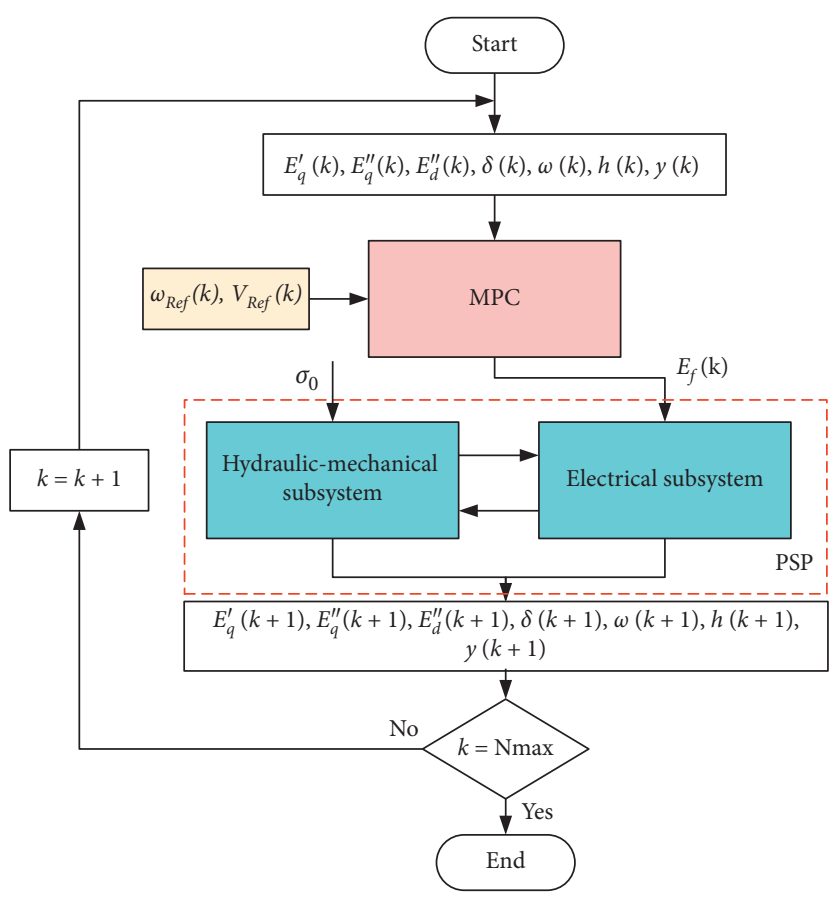

FIGURE 18: Flow charts of noncoupling MPC strategy.

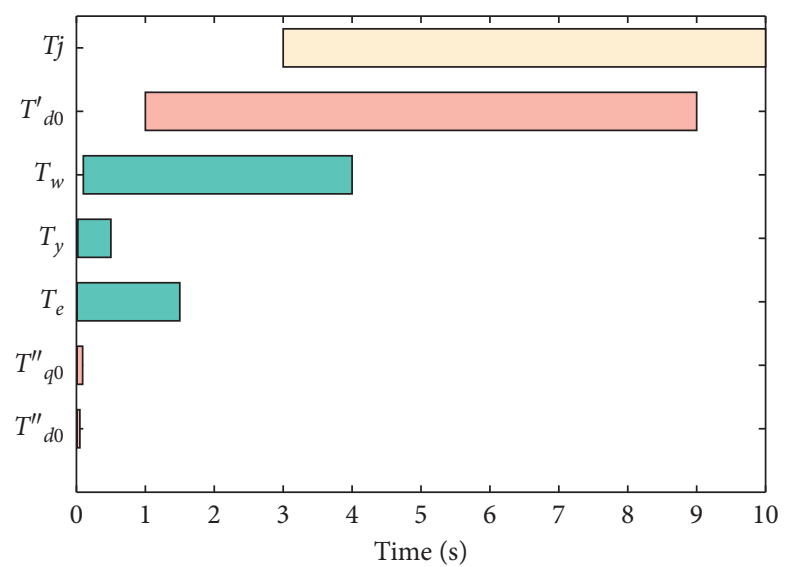

Figure 19: Common ranges of some basic time constants in PSP.
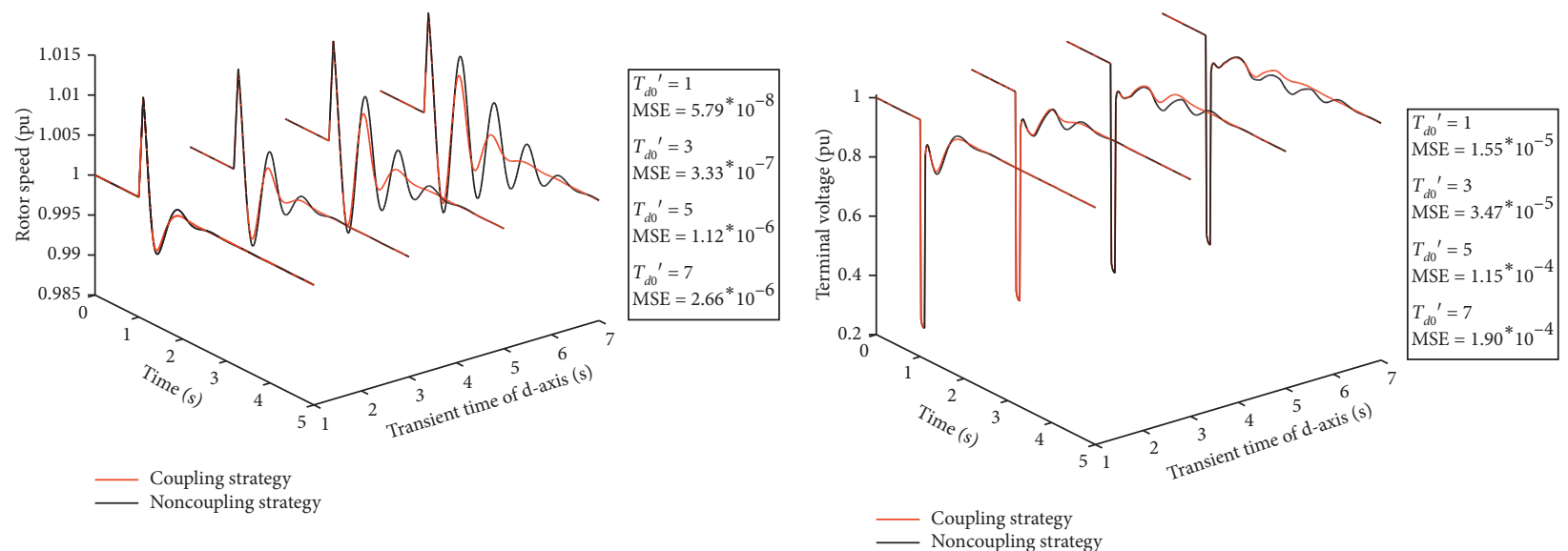

(a)

(b)

FIGURE 20: Response comparison under a three-phase short circuit fault condition of different transient time constants of the d-axis. (a) Rotor speed response. (b) Terminal voltage response. 
$T_{d 0}^{\prime}$ are shown in Figure 20. It is shown that with the increase of time constant $T_{d 0}^{\prime}$, the effect on control performance from coupling factors is becoming greater. This is because when the time constant $T_{d 0}^{\prime}$ is small, the regulation speed of the hydraulic-mechanical subsystem is much slower than that of the electrical subsystem.

Currently, even considering the regulation influence of the hydraulic-mechanical system is not effective for the control of the coupling system. But, when the constant time $T_{d 0}^{\prime}$ becomes larger, the response speed of the electrical subsystem is close to that of the hydraulicmechanical subsystem. Accordingly, the coupling influence from hydraulic-mechanical subsystem can be considerable. Considering the coupling effect of the hydraulic-mechanical-electrical system in this situation, the control quality can be greatly improved.

\section{Conclusions}

In this paper, an NMPC strategy is proposed based on the WOS-ELM prediction model and ASA rolling optimization for the hydraulic-mechanical-electrical coupling system of PSP. Specifically, the initial weights and the bias of the hidden layer in ELM, which can fully reflect the internal relationship of the modelling data, is optimized by ASA, while the output weights are still calculated by the least squares method. Besides, the ASA is also used in the rolling optimization mechanism of NMPC to replace the existing complex differential geometric solutions. In order to reduce the computational burden, the idea of stair-like control strategy is introduced into rolling optimization. What's more, the forgetting factor is adjusted adaptively according to the prediction error in the feedback revision.

The proposed MPC method has been verified and tested with comparative experiments, while the nonlinear simulation model based on a PSP in China is adopted as the study object. The conventional PI controller with AVR and PSS are compared with the proposed MPC method under diverse working conditions. Experimental results on the sudden step up in terminal voltage, three-phase short-circuit fault, and load adjustment conditions have proven the effectiveness and robustness of the proposed method. The results indicate that the proposed MPC strategy has superiority in control performance under uncertain conditions. Besides, the influence on control performance from coupling factors can be considerable, and the proposed MPC strategy, which takes the regulation effect of hydraulic-mechanical subsystem into account, has a significant advantage over the one that only considers the electrical effect.

\section{Abbreviations}

$\begin{array}{ll}E_{f}: & \text { Excitation EMF }(\mathrm{pu}) \\ E_{q}^{\prime}: & \text { Transient EMF of q-axis }(\mathrm{pu}) \\ E_{d}^{\prime \prime}: & \text { Sub-transient EMF of d-axis }(\mathrm{pu}) \\ E_{q}^{\prime \prime}: & \text { Sub-transient EMF of q-axis }(\mathrm{pu}) \\ f: & \text { Water head loss coefficient } \\ h: & \text { Water head }(\mathrm{pu}) \\ I_{d}: & I_{q} \text { Armature current of d- and q-axes }(\mathrm{pu})\end{array}$

$m_{t}: \quad$ Mechanical moment $(\mathrm{pu})$

$m_{11}$ : Unit mechanical moment (pu)

$n_{11}$ : Unit rotational speed (pu)

$P_{e}: \quad$ Electric active power $(\mathrm{pu})$

$P_{m}: \quad$ Mechanical power (pu)

$q$ : $\quad$ Discharge at pump-turbine (pu)

$q_{11}$ : Unit discharge (pu)

$T_{d 0}^{\prime}$ : Transient time constant of d-axis (s)

$T_{d 0}^{\prime \prime}$ : Sub-transient time constant of d-axis (s)

$T_{q 0}^{\prime \prime}$ : Sub-transient time constant of q-axis (s)

$T_{j}$ : Mechanical time constant (s)

$T_{e}$ : $\quad$ Reflection time of water hammer (s)

$T_{w}$ : Water starting time constant (s)

$T_{y}$ : $\quad$ Servo response time (s)

$V_{g}: \quad$ Voltage at the generator terminal (pu)

$V_{d} V_{q}$ : Terminal voltage of $\mathrm{d}$ - and q-axes (pu)

$V_{s}$ : Voltage of the infinite bus (pu)

$X_{d}: \quad$ Synchronous reactance of d-axis (pu)

$X_{d}^{\prime}$ : Transient reactance of $\mathrm{d}$-axis (pu)

$X_{d}^{\prime \prime}: \quad$ Sub-transient reactance of $\mathrm{d}$-axis $(\mathrm{pu})$

$X_{q}$ : Synchronous reactance of q-axis (pu)

$X_{q}^{\prime \prime}$ : $\quad$ Sub-transient reactance of q-axis (pu)

$X_{s}^{q}: \quad$ Total reactance of transmission line $(\mathrm{pu})$

$y: \quad$ Guide vane opening $(\mathrm{pu})$

$\sigma: \quad$ Input of servomechanism (pu)

$\delta$ : $\quad$ Rotor angle (rad)

$\omega$ : $\quad$ Rotor angular speed $(\mathrm{pu})$

$\omega_{0}$ : Synchronous angular speed $(\mathrm{rad} / \mathrm{s})$

$\Delta: \quad$ Deviation from the initial value.

\section{Data Availability}

The data used to support the findings of this study are not available because they involve the parameters and full characteristic data of the actual pumped storage power station.

\section{Conflicts of Interest}

The authors declare that they have no conflicts of interest.

\section{Acknowledgments}

This work was supported by the National Natural Science Foundation of China (NSFC) (Grant Nos.51809099, 51879111, and 51679095) and the Fundamental Research Funds for the Central Universities (Grant No. B210201010).

\section{References}

[1] D. Elliott;, "A balancing act for renewables," Nature Energy, vol. 1, p. 15003, 2012.

[2] A. S. Brouwer, "Operational flexibility and economics of power plants in future low-carbon power systems," Applied Energy, vol. 156, pp. 107-128, 2015.

[3] World energy Outlook 2018. https://www.iea.org/weo2018/.

[4] W. Deason, "Comparison of $100 \%$ renewable energy system scenarios with a focus on flexibility and cost," Renewable \& Sustainable Energy Reviews, vol. 82, pp. 3168-3178, 2017. 
[5] M. K. Chang, J. D. Eichman, F. Mueller, and S. Samuelsen, "Buffering intermittent renewable power with hydroelectric generation: a case study in California," Applied Energy, vol. 112, pp. 1-11, 2012.

[6] C. Su, C. Cheng, P. Wang, J. Shen, and X. Wu, "Optimization model for long-distance integrated transmission of wind farms and pumped-storage hydropower plants," Applied Energy, vol. 242, pp. 285-293, 2019.

[7] J. Hou, C. Li, W. Guo, and W. Fu, "Optimal successive startup strategy of two hydraulic coupling pumped storage units based on multi-objective control," International Journal of Electrical Power \& Energy Systems, vol. 111, pp. 398-410, 2019.

[8] Y. Xu, J. Zhou, X. Xue, W. Fu, W. Zhu, and C. Li, "An adaptively fast fuzzy fractional order PID control for pumped storage hydro unit using improved gravitational search algorithm," Energy Conversion and Management, vol. 111, pp. 67-78, 2016.

[9] C. Feng, L. Chang, C. Li, T. Ding, and Z. Mai, "Controller optimization approach using LSTM-based identification model for pumped-storage units," IEEE Access, vol. 7, pp. 32714-32727, 2019.

[10] J. Zhou, Y. Zhang, Y. Zheng, and Y. Xu, "Synergetic Governing controller design for the hydraulic turbine governing system with complex conduit system," Journal of the Franklin Institute, vol. 355, pp. 4131-4146, 2018.

[11] W. Yang and J. Yang, "Advantage of variable-speed pumped storage plants for mitigating wind power variations: integrated modelling and performance assessment," Applied Energy, vol. 237, pp. 720-732, 2019.

[12] M. Eremia and M. Shahidehpour, Handbook of Electrical Power System Dynamics: Modeling, Stability, and Control, John Wiley \& Sons, Hoboken, NJ, USA, 2013.

[13] W. Yang and J. Yang, "Evaluating fast power response of variable speed pumped storage plants to balance wind power variations," Energy Procedia, vol. 158, pp. 6341-6346, 2019.

[14] C. Li, N. Zhang, X. Lai, J. Zhou, and Y. Xu, "Design of a fractional-order PID controller for a pumped storage unit using a gravitational search algorithm based on the Cauchy and Gaussian mutation," Information Sciences, vol. 396, pp. 162-181, 2017.

[15] C. Li, Y. Mao, J. Zhou, N. Zhang, and X. An, "Design of a fuzzy-PID controller for a nonlinear hydraulic turbine governing system by using a novel gravitational search algorithm based on Cauchy mutation and mass weighting," Applied Soft Computing, vol. 52, pp. 290-305, 2017.

[16] W. Yang, P. Norrlund, C. Y. Chung, J. Yang, and U. Lundin, "Eigen-analysis of hydraulic-mechanical-electrical coupling mechanism for small signal stability of hydropower plant," Renewable Energy, vol. 115, pp. 1014-1025, 2018.

[17] W. Yang, P. Norrlund, J. Bladh, J. Yang, and U. Lundin, "Hydraulic damping mechanism of low frequency oscillations in power systems: quantitative analysis using a nonlinear model of hydropower plants," Applied Energy, vol. 212, pp. 1138-1152, 2018.

[18] J. Zhou, Z. Zhao, C. Zhang, C. Li, and Y. Xu, "A real-time accurate model and its predictive fuzzy PID controller for pumped storage unit via error compensation," Energies, vol. 11, p. 35, 2018.

[19] Y. Xu, Y. Zheng, Y. Du, W. Yang, X. Peng, and C. Li, "Adaptive condition predictive-fuzzy PID optimal control of start-up process for pumped storage unit at low head area," Energy Conversion and Management, vol. 177, pp. 592-604, 2018.
[20] C. Li, Y. Mao, J. Yang, Z. Wang, and Y. Xu, "A nonlinear generalized predictive control for pumped storage unit," Renewable Energy, vol. 114, pp. 945-959, 2017.

[21] K. Katić, R. Li, J. Verhaart, and W. Zeiler, "Neural network based predictive control of personalized heating systems," Energy and Buildings, vol. 174, pp. 199-213, 2018.

[22] F. Pazhooh, F. Shahraki, J. Sadeghi, and M. Fakhroleslam, "Multivariable adaptive neural network predictive control in the presence of measurement time-delay; application in control of Vinyl Acetate monomer process," Journal of Process Control, vol. 66, pp. 39-50, 2018.

[23] J.-H. Shin, H.-B. Jun, and J.-G. Kim, "Dynamic control of intelligent parking guidance using neural network predictive control," Computers \& Industrial Engineering, vol. 120, pp. 15-30, 2018.

[24] A. Pourfard, H. Moetamedzadeh, R. Madoliat, and E. Khanmirza, "Design of a neural network based predictive controller for natural gas pipelines in transient state," Journal of Natural Gas Science and Engineering, vol. 62, pp. 275-293, 2019.

[25] C. Zhang, T. Peng, J. Zhou, J. Ji, and X. Wang, “An improved autoencoder and partial least squares regression-based extreme learning machine model for pump turbine characteristics," Applied Sciences, vol. 9, no. 19, p. 3987, 2019.

[26] G.-B. Huang, Q.-Y. Zhu, and C.-K. Siew, "Extreme learning machine: theory and applications," Neurocomputing, vol. 70, no. 1-3, pp. 489-501, 2006.

[27] N. Y. Liang, G. B. Huang, P. Saratchandran, and N. Sundararajan, "A fast and accurate online sequential learning algorithm for feedforward networks," IEEE Transactions on Neural Networks, vol. 17, no. 6, pp. 1411-1423, 2006.

[28] P. K. Wong, H. C. Wong, C. M. Vong, Z. Xie, and S. Huang, "Model predictive engine air-ratio control using online sequential extreme learning machine," Neural Computing and Applications, vol. 27, no. 1, pp. 79-92, 2016.

[29] A. Mozaffari, M. Vajedi, and N. L. Azad, "A robust safetyoriented autonomous cruise control scheme for electric vehicles based on model predictive control and online sequential extreme learning machine with a hyper-level fault tolerancebased supervisor," Neurocomputing, vol. 151, pp. 845-856, 2015.

[30] H. Zhang, S. Zhang, and Y. Yin, "Online sequential ELM algorithm with forgetting factor for real applications," $\mathrm{Neu}$ rocomputing, vol. 261, pp. 144-152, 2017.

[31] Y. Xu, C. Li, Z. Wang, N. Zhang, and B. Peng, "Load frequency control of a novel renewable energy integrated micro-grid containing pumped hydropower energy storage," IEEE Access, vol. 6, pp. 29067-29077, 2018.

[32] Y. Xu, J. Zhou, C. Zhang, Y. Zhang, C. Li, and Z. Qian, “A parameter adaptive identification method for a pumped storage hydro unit regulation system model using an improved gravitational search algorithm," Simulation, vol. 93, no. 8, pp. 679-694, 2017.

[33] Z. Zhao, J. Yang, W. Yang, J. Hu, and M. Chen, "A coordinated optimization framework for flexible operation of pumped storage hydropower system: nonlinear modeling, strategy optimization and decision making," Energy Conversion and Management, vol. 194, pp. 75-93, 2019.

[34] J. Machowski, J. W. Bialek, and J. Bumby, Power System Dynamics: Stability and Control, John Wiley \& Sons, Hoboken, NJ, USA, 2011.

[35] X. Lai, C. Li, N. Zhang, and J. Zhou, "A multi-objective artificial sheep algorithm," Neural Computing and Applications, vol. 31, no. 8, pp. 4049-4083, 2019. 
[36] W. Wang, C. Li, X. Liao, and H. Qin, "Study on unit commitment problem considering pumped storage and renewable energy via a novel binary artificial sheep algorithm," Applied Energy, vol. 187, pp. 612-626, 2017.

[37] X. Lai, C. Li, J. Zhou, and N. Zhang, "Multi-objective optimization of the closure law of guide vanes for pumped storage units," Renewable Energy, vol. 139, pp. 302-312, 2019.

[38] Y. Zheng, J. Zhou, W. Zhu, C. Zhang, C. Li, and W. Fu, "Design of a multi-mode intelligent model predictive control strategy for hydroelectric generating unit," Neurocomputing, vol. 207, pp. 287-299, 2016.

[39] Y. Zheng, J. Zhou, Y. Xu, Y. Zhang, and Z. Qian, “A distributed model predictive control based load frequency control scheme for multi-area interconnected power system using discrete-time Laguerre functions," ISA Transactions, vol. 68, pp. 127-140, 2017.

[40] Z. Tao, W. Gang, H. Defeng, and Y. Dazhi, “An efficient model nonlinear predictive control algorithm based on stair-like control strategy," in Proceedings of the 2008 27th Chinese Control Conference, pp. 557-561, IEEE, Kunming, China, 2008.

[41] D. Q. Mayne, J. B. Rawlings, C. V. Rao, and P. O. M. Scokaert, "Constrained model predictive control: stability and optimality,” Automatica, vol. 36, no. 6, pp. 789-814, 2000. 